Revue scientifique sur la conception et l'aménagement de l'espace

\title{
Gouverner un territoire de l'eau à partir de considérations sur les savoirs liés à la production du paysage
}

Governing Waterscapes Based on Considerations of Knowledge of Landscape Planning

Jacques-Aristide Perrin

\section{(2) OpenEdition}

\section{Journals}

Édition électronique

URL : http://journals.openedition.org/paysage/615

DOI : $10.4000 /$ paysage.615

ISSN : 1969-6124

Éditeur :

École nationale supérieure du paysage de Versailles-Marseille, Institut national des sciences appliquées Centre Val de Loire - École de la nature et du paysage, École nationale supérieure d'architecture et de paysage de Bordeaux, École nationale supérieure d'architecture et de paysage de Lille, Agrocampus Angers

\section{Référence électronique}

Jacques-Aristide Perrin, « Gouverner un territoire de l'eau à partir de considérations sur les savoirs liés à la production du paysage », Projets de paysage [En ligne], 20 | 2019, mis en ligne le 17 juin 2019, consulté le 28 novembre 2019. URL : http://journals.openedition.org/paysage/615 ; DOI : 10.4000/ paysage.615

Ce document a été généré automatiquement le 28 novembre 2019.

Projets de paysage 


\section{Gouverner un territoire de l'eau à partir de considérations sur les savoirs liés à la production du paysage}

Governing Waterscapes Based on Considerations of Knowledge of Landscape Planning

Jacques-Aristide Perrin

1 Cet article s'intéresse à une politique publique environnementale dénommée la continuité écologique des cours d'eau (CECE). En analysant sa mise en œuvre lors d'opérations de terrain, il ambitionne de saisir la manière dont pourraient être mieux pris en compte les enjeux paysagers.

2 La CECE est considérée comme un des moyens pour atteindre le très bon état écologique des masses d'eau dans le cadre de la directive-cadre sur l'eau, votée en 2000, et de la loi sur l'Eau et les Milieux aquatiques de 2006. La politique publique de la CECE ambitionne de réunir les conditions adéquates pour favoriser la circulation des poissons (essentiellement migrateurs) et des sédiments dans des dimensions amontaval et latérale (berges). En plus de ces objectifs affichés de préservation de la biodiversité piscicole et d'amélioration de la qualité hydro-morphologique des cours d'eau, la CECE est également présentée par ses promoteurs (ministère de l'Environnement et ses services déconcentrés, agences de l'Eau, certaines associations environnementales) comme un moyen d'améliorer la qualité de l'eau. À ces fins, des opérations de terrain sont menées sur des ouvrages hydrauliques (seuils de moulins d'agrément ou producteurs d'énergie, prises d'eau à vocation agricole, petits barrages, digues, seuils de dérivation servant à l'hydroélectricité ou à des espaces de loisirs). Deux types de solutions sont principalement préconisés : soit ré-aménager l'ouvrage hydraulique visé par la construction de passes à poissons, d'un bras de contournement ou la réduction de la hauteur d'un ouvrage, soit supprimer cet ouvrage si les conditions sont réunies. Alors que des actions similaires ont été entreprises avec parcimonie en 
France dès les années 1980 (Germaine, Barraud, 2017) au nom d'une continuité piscicole, elles prennent aujourd'hui une autre ampleur en se déployant, cette fois, sur des milliers d'ouvrages hydrauliques depuis les années 2010.

Les projets de restauration de la CECE se situent au croisement de plusieurs préoccupations, à savoir la priorisation entre des patrimoines dits naturels et bâtis, des enjeux paysagers ainsi que des usages liés aux cours d'eau (moulins productifs comme un économusée, une minoterie ou une tannerie, moulins d'agréments, seuils de dérivation servant pour l'hydroélectricité, pour alimenter des canaux d'irrigation ou des plans d'eau destinés à la baignade, spots de pêche aux abords des ouvrages...). Or, son application s'effectue, pour certaines opérations, dans un contexte d'opposition avec la crainte, de la part des propriétaires d'ouvrages privés ou des riverains, de voir modifier les milieux aquatiques auxquels ils sont habitués et attachés. En effet, comme nous le verrons, les travaux peuvent entraîner des effets différents sur les paysages selon le type de solution choisie. Un rapport de force est alors parfois engagé entre les différentes parties prenantes pour défendre ce à quoi elles tiennent le plus.

4 S'il y a différentes manières d'aborder cet objet d'étude sous l'angle paysager selon les approches et méthodes de disciplines et métadiscipline avec la science du paysage (Bertrand, 1972) et les sciences de la durabilité, cet article entend s'inscrire en géographie politique de l'environnement pour étudier la manière dont la gestion des paysages de l'eau est cadrée par l'action publique de la CECE. Le cadrage des enjeux correspond à un " choix, pas toujours explicité, de ce qui est pris en considération et ce qui reste "hors-champ" dans le travail d'expertise " (Joly, 2012). En portant notre attention sur « les interactions entre les processus physiques, les pratiques matérielles, culturelles et les constructions idéologiques de la valeur de l'eau " (Blanchon, 2009, p.57), il s'agit de saisir comment des outils et les types de savoirs mobilisés dans l'action publique cadrent les enjeux sans que certaines considérations (goûts, représentations, intérêts vis-à-vis du patrimoine) soient abordées par les acteurs sur les territoires des eaux. Avec cette approche centrée sur les processus politiques de la production des paysages de l'eau, nous chercherons à montrer que les outils mobilisés pour légitimer et réaliser la CECE s'intéressent très peu aux paysages, dans leurs aspects aussi bien esthétiques que fonctionnels, alors même que penser en ces termes permettrait de sortir momentanément de logiques d'intérêt. De manière complémentaire à un écrit récent qui s'interrogeait sur le projet de paysage comme "alternative pour penser et agir sur la restauration écologique des cours d'eau " (Davodeau et Romain, 2017, p. 163), nous nous attachons particulièrement au rôle des savoirs liés à la production du paysage et à leurs détenteurs pour expliciter une demande des acteurs locaux de recourir à une diversité de savoirs (notamment historiques, multiscalaires et situés dans des espaces vécus).

Dans le cadre de cet article, nous ne sommes intéressé que par les rivières qui traversent des espaces habités et des territoires aux périmètres divers. En ce sens, les territoires de l'eau sont appréhendés de manière plurielle, à la fois du point de vue de la décentralisation institutionnelle (Ghiotti, 2006) sur le modèle du bassin-versant ou de celui de la "dimension culturelle propre à des pratiques et des représentations " (Alexandre et Arrus, 2004) correspondant à des enjeux liés aux bassins de vie et à l'identité partagée par les habitants. Les opérations d'application de la CECE prennent place dans des contextes territoriaux, institutionnels et scalaires différents. Plusieurs structures peuvent piloter des opérations comme des syndicats de rivière, des 
établissements publics territoriaux de bassin, des arcs naturels régionaux ou encore des fédérations départementales de pêche. Les opérations peuvent alors prendre un sens différent pour les habitants selon si cette structure gère la mise en œuvre sur un seul tronçon à restaurer, le cours d'eau entier ou les eaux d'un bassin-versant (qui peut traverser des limites administratives).

Pour étudier ces enjeux paysagers sur les territoires de l'eau, nous nous sommes appuyé sur des études de cas réalisées entre 2014 et 2017 en France. Nous avons suivi l'évolution d'opérations sur plusieurs rivières appartenant à deux bassins-versants différents. D’une part, le bassin de la Têt, situé dans les Pyrénées-Orientales, prend sa source dans une zone montagneuse pyrénéenne avant de traverser un secteur de plaine et une zone urbaine avec l'agglomération de Perpignan. Tandis qu'une minorité des ouvrages se trouve sur le fleuve Têt en aval, la majorité des ouvrages hydrauliques concernés par la CECE est installée dans la zone montagneuse sur les affluents du fleuve Têt et sont gérés par concession par un opérateur hydroélectrique. Étant donné la faible densité de population et les efforts nécessaires pour accéder à ces sites, les enjeux paysagers se situent davantage en aval du fleuve.

Figure 1. La Têt, axe central du département

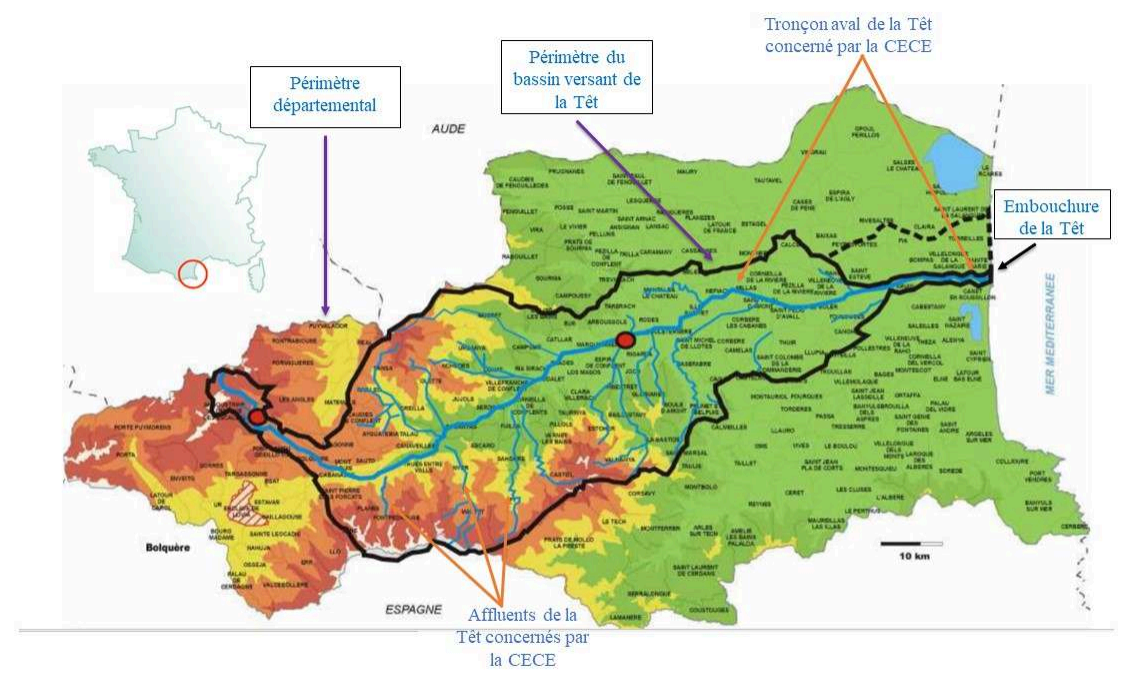

Fond de carte : Syndicat mixte Bassin Versant de la Têt.

D'autre part, nous nous sommes intéressés à cinq cours d'eau (la Bave, la Couze, la Dronne, le Mamoul, la Tude) dans le bassin-versant de la Dordogne qui sont soumis au droit privé de propriété des riverains. Ces cours d'eau s'inscrivent dans des territoires de l'eau habités, traversant des zones rurales et urbaines, donnant lieu à des enjeux différents en fonction des paysages, des usages et des formes d'attachement à des pratiques (pêche, canoë, baignade). 


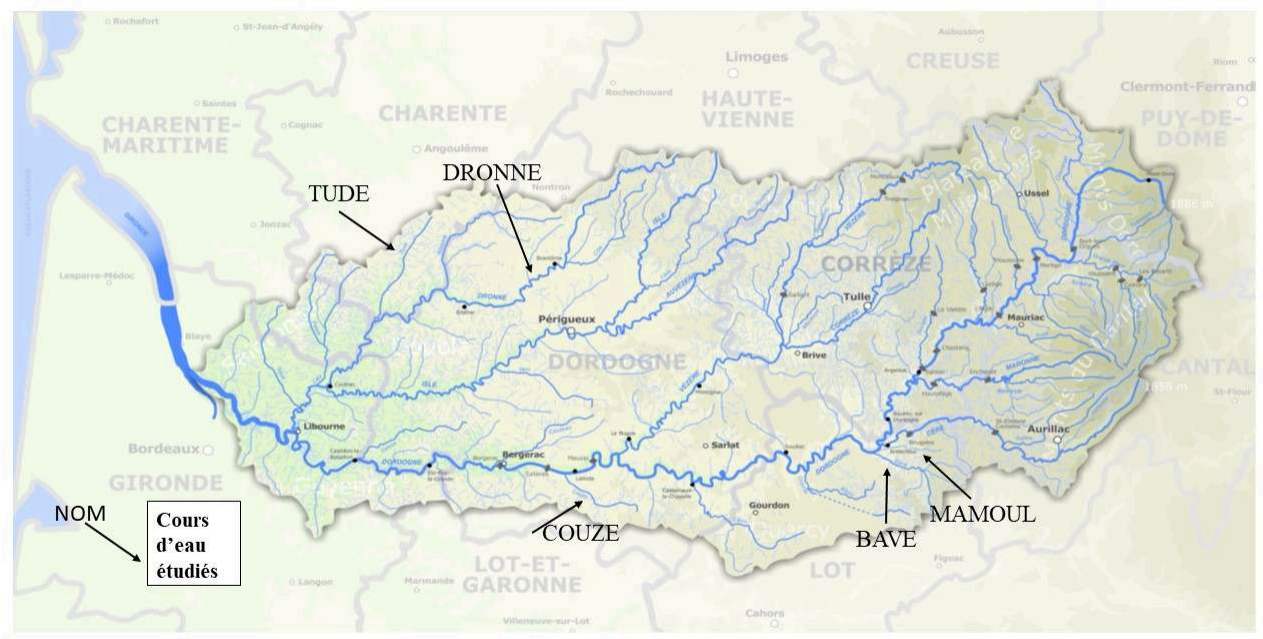

Fond de carte : La vallée de la Dordogne, EPIDOR, 2013.

Faisons remarquer qu'à l'exception de certains ouvrages sur la Têt, en paysage montagneux, l'essentiel des opérations de CECE étudiées a pris place dans des paysages de fond de vallée que l'on peut considérer comme des "paysages ordinaires " (Germaine, 2009) avec des petits et anciens ouvrages hydrauliques. Les enjeux en milieu très urbanisé disposent de leurs propres spécificités (Coninck, 2015). Les terrains d'étude nous ont amené à analyser des ouvrages publics, mis en concession et privés, tantôt à usage direct (hydroélectricité, seuil de dérivation pour un canal d'irrigation), tantôt à usage indirect (aménité paysagère), sans usage ou laissés à l'abandon. Nous nous servons ainsi de l'ensemble des cas d'étude (aux caractéristiques sociopolitiques, culturelles et biogéophysiques propres), sans les détailler un à un avec minutie, pour faire ressortir les éléments explicatifs généraux des difficultés de la mise en œuvre de cette politique.

Enfin, notre méthodologie de travail a consisté à suivre les parties prenantes durant les procédures prévues par la politique publique qui, souvent, prennent place en parallèle des outils des schémas d'aménagement et de gestion de l'eau. Les étapes de la procédure de CECE, fixées par la loi, sont les suivantes: prise de contact par courrier avec les propriétaires pour les informer de la nécessité de mettre en conformité leurs ouvrages avec la loi, organisation de réunions d'information, négociation concernant les clauses des études avec le bureau d'études, réalisation de l'étude sur chaque ouvrage hydraulique avec une présentation des scenarii possibles, décision du propriétaire sur la solution à appliquer, lancement des travaux, éventuel suivi des effets des travaux. En complément à cette observation, nous avons réalisé une quarantaine d'entretiens semidirectifs avec les parties prenantes des opérations étudiées. Les acteurs interviewés comprennent 14 propriétaires et gestionnaires d'ouvrages, 10 riverains, 4 acteurs de la pêche, 3 agents de bureau d'études, 15 acteurs publics (État et collectivités territoriales), encadrant et pilotant dans les procédures d'application. Ces entretiens, analysés textuellement, ont été pensés autant pour récolter des données qui sont assez peu nombreuses dans les documents administratifs - recueillir les points de vue des acteurs concernant la manière dont est appliquée la CECE sur leurs territoires -, que 
pour identifier les savoirs qui leur permettent de juger du bien-fondé de cette politique publique.

Dans un premier temps, nous reviendrons sur les changements paysagers que la CECE peut engendrer. Les craintes et les effets réels de son application favorisent alors l'émergence de contestations. Dans un second temps, nous montrerons que les difficultés d'intégrer une diversité d'enjeux en raison du cadrage de la CECE peuvent être surmontées en réfléchissant aux types de savoirs à mobiliser en lien avec les paysages des territoires. L'approche paysagère, à l'interstice des enjeux sociopolitiques et sociotechniques, peut représenter un outil propice à la construction stratégique des territoires de l'eau dans le cadre de pratiques de gouvernement.

\section{Enjeux paysagers de cette politique publique des eaux}

Dans les paysages de l'eau, la place simple ou dominante de cet élément est un sujet de discussion régulier lors de projets d'aménagement. Avec la politique de la CECE, telle que conçue dans les années 2000 durant l'élaboration de la loi sur l'Eau et du Grenelle de l'environnement $\mathrm{n}^{\circ} 1$ (Perrin, 2018), la longitudinalité des rivières est placée au centre des attentions. Elle devient une composante à respecter en tant que telle, au nom des circulations piscicoles et sédimentaires, qui s'impose à d'autres composantes du paysage comme les ouvrages hydrauliques à usages directs ou indirects. Sur un plan juridique, il s'agit d'ailleurs d'une évolution notable puisque, auparavant, un aménagement dans les eaux devait passer par une autorisation administrative démontrant que les dommages collatéraux sur la continuité des eaux pouvaient être minimisés et compensés. Désormais, les aménageurs doivent prouver que les avantages qu'ils peuvent retirer des eaux sont suffisamment importants au point de justifier un ébrèchement de la continuité des cours d'eau. L'avènement de la CECE a ainsi introduit une inversion de la charge de la preuve. Comme nous le verrons, cette inversion d'origine axiologique n'est pas sans poser problème quant à la manière d'intégrer les cours d'eau dans un projet de territoire qui prenne en compte l'histoire des relations entre les eaux et les habitants.

\section{Types de modifications sur les paysages de l'eau}

Le projet de la CECE consiste en une mise à l'épreuve des rivières et des paysages de l'eau désirés avec un choix à effectuer entre des rivières ensauvagées, partiellement restaurées selon l'état de référence naturel sélectionné, et des rivières qui demeurent aménagées (Germaine, Barraud, 2013). La décision, quant à la manière d'appliquer la CECE, pose ainsi des enjeux territoriaux avec des modifications d'usages (directs et indirects), questionnant sur la forme et le fond l'orientation des mutations paysagères (Rivière-Honegger, 2008) et la composition des sociomilieux aquatiques.

Ces changements dépendent premièrement de la solution choisie par un propriétaire/ gestionnaire d'ouvrage privé ou public, avec plusieurs facteurs à prendre en compte : intention au départ de la procédure, prise de connaissance du taux de financement disponible pour l'accompagner dans la réalisation des études et des travaux, éventuelle pression relayée durant la procédure d'application de la politique publique, présentation des coûts et des effets concrets des solutions techniques proposées. Bien que cette décision repose théoriquement sur le seul choix du propriétaire/gestionnaire, 
des enjeux structurants pour un territoire peuvent conditionner la décision finale selon leurs degrés d'importance. Il peut s'agir de la présence d'une espèce plus ou moins prestigieuse à préserver comme le saumon atlantique, de la production d'hydroélectricité pourvoyeuse en emplois, de la valeur patrimoniale et foncière d'un ouvrage productif ou d'agrément. Chaque décision n'est finalement pas purement autonome puisque les propriétaires d'un même tronçon peuvent se concerter et les décisions peuvent devenir interdépendantes. Les opérations de la CECE peuvent avoir des répercussions à différentes échelles (celles d'un site, d'un tronçon de rivière, voire d'une rivière) avec des effets bio-géo-physiques sur l'hydrosystème en question et la vie aquatique, mais également en matière d'image territoriale lorsqu'une collectivité territoriale entreprend des actions volontaristes sur le plan environnemental. Cela fut le cas sur un de nos terrains d'étude, avec le Parc naturel régional Périgord-Limousin qui réalisa la CECE sur la Dronne en tant que prélude à une future labellisation « rivière sauvage ».

Modifier ou détruire un ouvrage hydraulique pour réaliser la CECE peut bouleverser le paysage local. Étant donné la variabilité de chaque site où peuvent être installés les seuils de rivière et de barrages, nous rendons compte de ces modifications (parfois interdépendantes) en les présentant avec la typologie suivante :

- espèces aquatiques à conserver: poissons grands migrateurs (anguille, grande alose, lamproie fluviatile, saumon atlantique), poissons migrateurs sur de plus petites distances (truite fario), poissons relativement sédentaires (brochet, perche, gardon, goujon), autres espèces aquatiques moins visibles mais dépendantes des espèces précédemment citées ou d'une continuité sédimentaire (moule perlière, écrevisse à pieds blancs) ;

- éléments à considérer: berge avec sa ripisylve, bief, bras de contournement, canal d'irrigation, canal d'amenée, chute, digue, fossé de drainage, grille et turbine pour l'hydroélectricité, infrastructure hydraulique, ligne d'eau, passe à poissons, plan d'eau, types d'arbres (aulne, frêne, tilleul...) qui bénéficient ou non des modifications ;

- caractéristiques de ces éléments qui peuvent fluctuer: hauteur (de la ligne d'eau, d'un ouvrage, de la berge...), forme (ouvrage hydraulique transversal, de biais, rectiligne), matériau (béton, roche, bois), couleur, son (chute d'eau, écoulement), espèces aquatiques (jugées prestigieuses, endémiques ou invasives), usages directs (productifs, intérêts économiques) ou indirects (agréments, loisirs comme la baignade) ;

- lieux d'accès à conserver ou non : sentier de promenade, spot d'observation selon le point de vue et le moment (de la faune et de la flore des milieux aquatiques, du paysage de l'eau), spot de pêche.

15 Ce qui peut être modifié dans le paysage relève tantôt de micro- ou de macroformes (Reyt, 1997), selon l'ampleur des changements, leurs interdépendances et les échelles auxquelles se référer pour les constater. De fait, pour appliquer la CECE, la décision finale fait intervenir des questionnements sur d'éventuelles restrictions d'usages. La gestion des usages demeure d'ailleurs le premier sujet de discussion entre les parties prenantes, en plus du coût de l'opération (souvent compris entre une dizaine de milliers d'euros et plusieurs centaines, selon le type d'ouvrage, la solution préconisée, le nombre d'espèces piscicoles à considérer et le débit du cours d'eau en question). Ainsi, malgré l'envergure de possibles modifications de micro- ou macroformes, l'enjeu paysager de la CECE n'est pas prioritaire dans le cadrage de cette politique et reste à la marge pour les acteurs contestataires comme nous allons le voir. 


\section{Une lutte de patrimonialisation entre des usages et des entités humaines/non humaines composant les eaux des territoires} usagers directs (hydroélectriciens, irrigants, moulins producteurs) ou indirects (kayakistes, pêcheurs de poissons blancs tels que le brochet et la perche considérés comme sédentaires, propriétaires d'ouvrages attachés à leurs patrimoines inactifs et aux paysages afférents) des cours d'eau. Le mécontentement de ces usagers est d'ailleurs souvent appuyé par celui des riverains. Deux types d'acteurs locaux se mobilisent principalement sur les territoires étudiés pour faire reconnaître la priorisation du patrimoine bâti sur celui biologique.

Il s'agit d'abord de certains adeptes de la pêche qui mettent en avant un changement de faciès d'écoulement des eaux. En effet, des pêcheurs en milieux lentiques (faible courant d'eau) craignent que leurs conditions de pêche se détériorent. Ce changement se concrétiserait par une nouvelle physionomie du tronçon de cours d'eau avec un courant plus rapide que celui dont ils sont habitués. Cette modification spatiale du (socio-) milieu aquatique est de fait discutée eu égard à l'attachement d'une partie des pêcheurs à leurs lieux de pratique de pêche. Cette situation se produit notamment sur la Tude. Les pêcheurs (380 cartes de pêche, tout type compris, par an en moyenne) craignent que la suppression d'ouvrages vienne réduire le débit moyen alors même que des cas de sécheresse ont été, durant ces dernières années, recensés. De plus, les meilleurs spots de pêche se situent à proximité des retenues d'eau. La réalisation de la CECE leur fait aussi craindre l'arrivée du silure (espèce dite invasive qui prolifère dans les eaux de la Dordogne). Compte tenu de leurs goûts et de leur résistance à « imaginer l'évolution voulue des paysages" (Germaine, 2011), ces pêcheurs préfèrent donc s'appuyer "sur le maintien des éléments hérités " (ibid.). Leurs revendications sont ainsi territoriales pour continuer à disposer d'espaces appropriés qui soient conformes à leurs désirs, pratiques et goûts esthétiques. Faisons remarquer que les pêcheurs mécontents semblent être une minorité parmi les pêcheurs puisque les fédérations départementales de pêche soutiennent la politique publique.

Quant aux propriétaires des ouvrages hydrauliques, ils s'opposent à une restriction de leur usage, voire à une demande de non-usage du cours d'eau. Les propriétaires sont sensibles à leurs seuils, même s'ils sont non productifs pour une grande majorité de cas étudiés, en raison de l'histoire qu'ils représentent (legs familiaux fréquemment). Pour les ouvrages encore productifs, la CECE questionne de surcroît l'activité de petites exploitations (sur la Dronne et la Bave : une tannerie, une minoterie, un économusée, une papeterie) et la rentabilité des micro- et picoproducteurs hydroélectriques. Les projets d'installation d'équipements de production d'énergie pour le présent ou le futur, au regard de la valeur d'option des ouvrages, sont également remis en cause avec la CECE. En outre, les propriétaires mettent en avant un risque pour la valeur foncière de leurs moulins en tant que résidences principales ou secondaires, lieux servant de gites et de chambres d'hôte. En cas de modification de l'ouvrage et du paysage d'agrément rattaché au cadre de vie (ripisylve maîtrisée, nuances végétales des bandes enherbées qui enserrent le canal et les rives, bruit de l'eau), les propriétaires craignent aussi une dépréciation de leurs biens et de la perte du droit d'eau.

19 Dans les discours de défense des biens patrimoniaux, nous avons distingué deux manières d'employer la notion de patrimoine. Pour les propriétaires d'ouvrages,

Projets de paysage, 20 | 2019 
essentiellement de moulins, il s'agit de sauvegarder les ouvrages qui représentent à la fois beaucoup pour leurs histoires personnelles et pour leurs territoires : un ouvrage est l'héritage de l'ancienneté d'un usage, d'un métier, d'un savoir-faire, d'une pratique ancestrale. Porte-parole de leurs patrimoines non productifs, ces opposants à l'application de la CECE demandent à les pérenniser avec néanmoins deux variantes dans les discours : certains en font la demande pour leurs usages purement personnels quand d'autres mettent en avant leurs utilités culturelle et historique. Par exemple, un représentant d'associations de moulin nous a expliqué qu'«il faut qu'il y ait un but. Il faut savoir être pragmatique en pareille occasion. L'organisation des journées du patrimoine de pays et des moulins est une opportunité pour montrer la valeur des moulins ${ }^{1}$ » sur un plan pédagogique. Inversement, les acteurs favorables à l'application de la CECE privilégient le patrimoine naturel d'ordre biologique/génétique : les espèces menacées doivent être la priorité à la fois pour leur survie et pour ce qu'elles permettent de dire de la qualité de l'eau. Les poissons migrateurs sont en effet considérés comme des espèces sentinelles et parapluies qui informent de l'état général de la qualité des eaux. Dans les deux cas, se produit une mise en patrimoine, qu'il convient de transmettre aux générations futures. En cela, «la patrimonialisation consiste en une "filiation inversée" : ce sont les acteurs du présent qui désignent dans le passé, en l'interprétant sur la base de critères contemporains, ce qu'ils estiment être leur tradition ou leur patrimoine. Cette "reconnaissance de paternité" (Lenclud) signifie que la patrimonialisation, loin de constituer le figement d'une tradition, a un caractère productif » (Babou, 2015).

Cette lutte pour déterminer le type de patrimoine à protéger est par conséquent productive socialement. Une mise en œuvre difficile de la CECE peut donner à voir des formes différentes de territorialisation selon le type de patrimoine devenu dominant au fil des échanges et des relations de pouvoir entre les acteurs. Cela a été valable sur toutes les opérations étudiées à l'exception d'une seule. Les ouvrages hydrauliques en amont du bassin de la Têt n'ont pas donné lieu à de tels débats en raison de la faible densité de population sur ces espaces mais surtout le concessionnaire de ces ouvrages souhaitait conserver de bonnes relations avec les acteurs clefs (élus, collectivités, syndicat de gestion, associations...) dans un contexte de re-négociation des concessions hydroélectriques. Cette "posture ubiquiste» (Massardier, 2009) consista en des arrangements mutuels entre l'opérateur et les acteurs de terrain (fédération de pêche départementale notamment) sans que des discussions sur le rôle de la CECE pour le territoire départemental n'aient été entreprises. Ainsi, un enjeu plus structurant d'ordre national a conditionné la manière de répondre de cet opérateur face à la CECE.

21 Finalement, l'avènement de cette politique publique questionne le bien-fondé des usages des ouvrages hydrauliques et leurs effets sur les sociomilieux aquatiques. Les questionnements paysagers restent au second plan mais sont malgré tout présents, suite aux demandes des propriétaires, des usagers et des riverains d'en tenir compte. L'importance accordée par les propriétaires d'ouvrages à la présentation de plusieurs scenarii durant les études techniques en témoigne. Cela leur permet d'évaluer les effets (visuels) des travaux sur le paysage et la végétation d'un site dans une optique d'intégration paysagère des futurs travaux. 


\section{Les savoirs liés aux paysages comme méthode pour gouverner}

\section{Dépasser le cadrage de cette politique publique pour mieux penser ses fins et moyens}

La difficulté d'appréhender l'ensemble des enjeux et les effets des éventuelles modifications sur les paysages de l'eau démontre que la CECE n'est pas seulement une politique qui peut être circonscrite aux seules longitudinalité et latéralité des cours d'eau. Les goûts, intérêts et pratiques des usagers, des habitants et des riverains sont questionnés, ouvrant la voie à des réflexions sur les relations entre les eaux et les territoires. Décrites par la littérature scientifique (Barraud, 2007; Barraud, 2011; Le Calvez, 2017), ces situations de mécontentements et de conflits sur le terrain, relayées notamment par plusieurs canaux ${ }^{2}$ à l'échelle nationale, amènent les contestataires à remettre en cause la manière choisie pour répondre à l'enjeu de la CECE. Ils souhaitent que l'histoire locale et les enjeux spatiaux locaux dont découlent leurs pratiques (baignade, pêche, promenade...), habitudes et goûts (sonores, visuels, esthétiques) intervenant dans la composition des paysages de l'eau, soient mieux pris en compte.

En retraçant l'histoire de cette politique publique (Perrin, 2018), l'accent mis sur certaines composantes du paysage peut être expliqué grâce à une identification des savoirs clefs mobilisés dans l'action publique. Fidèle à la directive-cadre européenne dans laquelle s'inscrit l'application de la CECE, une approche a été choisie pour observer et agir sur les milieux dits naturels. Les activités anthropiques étant considérées comme des pressions sur le vivant, les experts ont sélectionné des savoirs qui leur ont permis dès lors de déployer tout un dispositif centré sur les seuils en tant qu'obstacle à la circulation. Le résultat des expertises successives (pour le compte de la Commission européenne, du ministère de l'Environnement et de ses agences) constitua un savoir-faire ingénieriste qui s'intéressa seulement à quelques enjeux liés aux ouvrages et aux espèces des grands migrateurs, en faisant abstraction d'autres enjeux comme les poissons ordinaires ou encore les effets de la destruction d'un seuil sur les rives. Les dimensions socioculturelles n'ont pu être traitées, l'ouverture aux enjeux paysagers ayant été écartés. Au demeurant, l'importance du savoir est prépondérante dans les opérations de restauration de la CECE car il a servi aussi bien à concevoir l'action publique (identification des modifications attendues et des moyens pour y parvenir) qu'à déterminer la manière dont elle devait être conduite durant les opérations.

Trois types de remises en cause liées aux savoirs mobilisés dans l'action publique sont principalement formulés. Premièrement, les savoirs et les outils d'action publique (indicateurs) sont contestés et jugés comme non adaptés à la réalité des relations hydrosociales. Les opposants mettent en avant la difficulté de sortir du monopole du cadrage de l'expertise officielle (en termes de disciplines, d'échelles d'analyse, d'approches pour connaître les milieux...), amenant à considérer seulement certains effets de la CECE sur la composition des cours d'eau. En effet, les choix d'échelles, d'approches (Bouleau et Pont, 2014) et d'indicateurs centrent particulièrement l'attention sur les ouvrages hydrauliques, en mettant de côté d'autres caractéristiques des milieux aquatiques, comme l'ouverture aux espèces invasives, la circulation de poissons, ou des répercussions non prévues sur les espèces piscicoles et végétales. Une 
équipe de chercheurs (Dufour et al., 2017) a montré que chaque échelle recouvre des considérations distinctes : ils mettent l'accent sur les effets de la CECE du point de vue $\mathrm{du}$ fonctionnement global de la plaine alluviale, dimension peu ou pas présente selon le cadrage de la CECE longitudinale. Ouvrir le champ d'observation à d'autres échelles, éléments du paysage et facteurs dans le temps peut donner une perspective différente des conséquences habituellement présentées. Telle que conçue, la CECE laisse penser qu'il y aurait une seule manière d'agir pour sauver les poissons migrateurs et améliorer la qualité de l'eau alors même que celle-ci est justement réexaminée par des propriétaires et des riverains. Ce travail critique sur les sciences mérite d'être mis à profit et a inspiré la proposition présentée en fin d'article.

Deuxièmement, la finalité du projet est discutée avec une question déterminante: quelles eaux et espèces sur les territoires sont désirées? Les opérations de la CECE ambitionnent principalement de sauver certaines populations piscicoles (grands migrateurs) auxquelles les acteurs publics et des associations environnementalistes attachent une importance particulière pour des raisons à la fois axiologiques (valeur symbolique d'un point de vue historique et génétique) et écologiques (espèces sentinelles de la qualité de l'eau qui sont des ambassadeurs des efforts entrepris depuis trente ans pour lutter contre le déclin de la biodiversité piscicole). Or, ces choix de valeurs et cette priorisation ne font pas nécessairement consensus sur tous les territoires de l'eau. Tantôt les propriétaires d'ouvrages acceptent majoritairement de participer à cet effort en faveur de la biodiversité, tantôt ils refusent cette priorisation des enjeux en faisant valoir d'autres priorités (conservation du patrimoine bâti et maintien de production d'énergie avec la force des eaux au nom d'une continuité sociohistorique et identitaire) et certains risques (effets incertains sur les berges, difficulté d'entretien des passes à poissons lorsque le courant est puissant, remobilisation de sédiments pollués dans les cas d'effacement d'ouvrage). Il y a ainsi un entremêlement d'enjeux politiques et scientifiques qui, selon le cadrage, peut orienter l'action publique. En outre, en mettant peu en avant la composition des paysages, le cadrage actuel court le risque de prédéterminer les éléments paysagers des fonds de vallée et, en définitive, d'homogénéiser les espaces attenants aux eaux (profils longitudinaux, types de végétalisation, structures des passes à poissons, choix des espèces vivantes à sauvegarder). La CECE engage effectivement un "modèle paysager de référence [...] pour dessiner la forme archétypale d'une rivière écologiquement en bon état. Celle-ci prend la forme d'une rivière à méandrage actif et banc de gravier " (Bercovitz, 2016).

Enfin, le niveau de décision est également discuté par les acteurs opposés à la CECE. Vivant sur les territoires dans lesquels se situent les tronçons de cours d'eau à restaurer, ils se sentent dépossédés du devenir des eaux de leurs territoires. Ils se mobilisent pour éviter que le cadrage des procédures, construites par des savoirs, ne devienne trop contraignant, ce qui les empêcherait de décider pleinement du devenir des eaux. Un tel débat renvoie à la tension entre l'intérêt général et sa traduction sur les territoires mais aussi à la démocratisation des sciences qui accompagne la prise de décision. Jouer sur les arguments d'autorité peut conduire à succomber à la "tentation de la boîte noire - sur le mode "c'est de la science, il n'y a rien à négocier" " (Barbier et Larrue, 2011). Cette difficile conciliation entre des préoccupations globales et des attentes locales n'est en rien spécifique à la CECE (Larrère, 1997). À l'époque de sa négociation, toutes les parties prenantes n'ont pas été conviées autour de la table. Cela explique pourquoi les enjeux locaux (d'attachement paysager/patrimonial, de veille 
pour voir l'évolution du cours d'eau, de gestion des berges) aient été relégués au second plan. Ce faisant, ces conditions n'ont pas été créées pour intégrer, dans les projets de territoire, les enjeux liés aux eaux.

Sortir de ces remises en cause pour penser les cours d'eau dans toutes leurs composantes pourrait passer par une approche paysagère en tant qu'outil de négociation (Battais et al., 2011) entre des enjeux sociopolitiques (valeurs, histoires, usages) et sociotechniques (savoirs et techniques à utiliser) pour œuvrer à la réalisation de projets de territoires cohérents.

\section{Une connectivité dans les territoires pour penser une mutation paysagère}

Partant de ces vifs débats sur les savoirs au cœur de l'action publique, l'entrée paysagère peut faire le pont entre le recours aux sciences et les enjeux d'usage sur un territoire donné. L'entrée par les savoirs liés à la production des paysages de l'eau n'est pas une demande explicitée de la part des propriétaires d'ouvrages et des riverains rassemblés contre l'application de la CECE. Néanmoins, cette proposition tient à un constat effectué suite à l'analyse des cas d'étude.

Les acteurs locaux regrettent que leurs connaissances situées ne soient pas mieux prises en compte dans les études réalisées par les bureaux d'études et les négociations avec les services techniques de l'État. Leurs situations vécues ne sont ainsi pas suffisamment valorisées de leurs points de vue. En réponse, les acteurs publics mettent en avant que les propriétaires et les riverains n'assurent pas assez leurs devoirs pour gérer les ouvrages et les rives mais relèvent aussi leurs déficiences de connaissances pour appréhender l'ensemble des enjeux de cette politique. De fait, le rôle des connaissances et des savoirs est devenu, au fil des procédures de la CECE, un enjeu dominant dans les discussions des parties prenantes. Les uns dénoncent une asymétrie de savoirs au profit du cadrage de l'action publique quand les autres parlent d'un niveau de connaissances nécessaire pour qu'ils puissent être légitimes à exprimer leur point de vue. Le tableau ci-dessous rend compte des différences de discours liées aux savoirs et aux connaissances.

Figure 3. Des discours aux éléments de savoirs bien différents

\begin{tabular}{|ll|l|l|}
\hline $\begin{array}{l}\text { Éléments de } \\
\text { savoirs liés au } \\
\text { paysage }\end{array}$ & $\begin{array}{l}\text { Savoirs mobilisés par les } \\
\text { acteurs pro-CECE }\end{array}$ & $\begin{array}{l}\text { Savoirs mobilisés par les } \\
\text { acteurs plus critiques }\end{array}$ \\
\hline $\begin{array}{l}\text { Type de savoir à académique } \\
\text { invoquer }\end{array}$ & $\begin{array}{l}\text { Savoir } \\
\text { hydromorphologie, } \\
\text { hydrobiologie), savoir d'ingénierie } \\
\text { pour les passes à poissons }\end{array}$ & $\begin{array}{l}\text { Savoirs d'usage (relatifs aux maniements } \\
\text { des vannes et à la gestion de passes à } \\
\text { poissons existantes), savoir sur l'histoire } \\
\text { locale des eaux }\end{array}$ \\
\hline $\begin{array}{l}\text { Mode } \\
\text { connaissance }\end{array}$ & $\begin{array}{l}\text { Mesure, } \\
\text { cartographie }\end{array}$ & $\begin{array}{l}\text { Observation vécue, pratique située, } \\
\text { archives }\end{array}$ \\
\hline
\end{tabular}




\begin{tabular}{|ll|l|l|}
\hline $\begin{array}{l}\text { Approche } \\
\text { milieu }\end{array}$ & du & $\begin{array}{l}\text { Pression des activités anthropiques } \\
\text { sur le milieu }\end{array}$ & $\begin{array}{l}\text { Pression des ambitions } \\
\text { environnementales sur le bâti et les } \\
\text { usages }\end{array}$ \\
\hline $\begin{array}{l}\text { Sujets } \\
\text { préoccupation les } \\
\text { plus invoqués }\end{array}$ & $\begin{array}{l}\text { Qualité des sédiments, circulation } \\
\text { des poissons grands migrateurs, } \\
\text { obstacle hydraulique comme }\end{array}$ & $\begin{array}{l}\text { Poissons plus ordinaires, circulation des } \\
\text { pollutions et des espèces invasives, } \\
\text { paysage d'un site, droit d'usage }\end{array}$ \\
\hline $\begin{array}{l}\text { Éléments } \\
\text { paysage } \\
\text { revendiqués }\end{array}$ & du & $\begin{array}{l}\text { Bras de contournement, lames } \\
\text { d'eau continue, passes à poissons }\end{array}$ & $\begin{array}{l}\text { Berge, ripisylve, bief, canal d'amenée, } \\
\text { chute d'eau, grille et turbine pour } \\
\text { l'hydroélectricité, plan d'eau }\end{array}$ \\
\hline Évaluation & Suivides populations piscicoles & $\begin{array}{l}\text { Effets sur les berges et la cinétique des } \\
\text { crues }\end{array}$ \\
\hline
\end{tabular}

Les acteurs ne s'expriment pas toujours avec les mêmes éléments de savoirs. Ils ne font également pas systématiquement référence aux mêmes enjeux ni de la même manière. Dans le cas des opérations les plus conflictuelles durant lesquelles aucune avancée notable n'a été possible, relancer les discussions sur les savoirs liés au paysage présente plusieurs avantages. En effet, comme le révèle Rémi Bercovitz dans sa thèse : «Le paysage a cette capacité à mettre à jour des liens d'interdépendance [...]. Inventer le paysage dans la perspective de la médiation, c'est rendre possible l'élaboration de significations et de représentations partagées ».

31 L'entrée paysagère permet autant d'explorer visuellement les pratiques d'aménagement et leurs effets sur l'environnement que de discuter des différents savoirs (expertises légitimées, locales) pour produire les paysages. L'ouverture du cadrage initial pourrait ainsi permettre de rediscuter de sujets sensibles, à l'instar de ce qui mérite d'être considéré comme un patrimoine (matériel, immatériel, non humain). L'expérience doctorale réalisée par Amandine de Coninck, pour évaluer les effets de différents scenarii sur la prise de décision collective concernant la CECE, pourrait être adaptée aux dimensions paysagères. Il s'agirait alors de modéliser différents scenarii selon des configurations d'éléments de savoirs liés à la production des paysages en tant qu'outil méthodologique de discussion. En tant que moyens de l'action, les savoirs liés au paysage participeraient à la formulation de diagnostics partagés et à la formulation de réponses spatiales à différentes échelles. Il convient néanmoins de se méfier d'un écueil principal : la compréhension et la production des paysages des eaux ne peuvent se réduire à une lecture du paysage essentiellement basée sur le visible, l'empirisme et le sensible. D'où la nécessité d'adjoindre à la médiation paysagère des savoirs issus d'horizons pluriels pour ne pas reproduire, comme avec la CECE, une simplification des hydrosystèmes anthropisés.

L'entrée par le paysage semble pouvoir constituer une alternative au cadrage de la CECE pour intégrer des enjeux sociopolitiques et sociotechniques à même de réfléchir aux enjeux sociopolitiques et sociotechniques qui permettront de déterminer les eaux qu'il est possible d'obtenir. Dans l'optique de travailler à des projets de territoire (Germaine et Lespez, 2014) en lien avec les eaux, il s'agit de tenir compte d'enjeux divers comme la préservation de la biodiversité, le cadre de vie et paysager, les stratégies de développement touristique élaborées sur un type de patrimoine, la 
production d'énergie, l'agriculture ou encore les risques environnementaux. À cette fin, une lecture pluriscalaire est un prérequis. Mettre en place une lecture multiscalaire des enjeux à l'échelle territoriale ne peut passer que par une connexion des différentes dimensions (longitudinale, latérale, verticale et temporelle) de l'hydrosystème, des vies non humaines avec les activités humaines pour entreprendre une perspective de gouvernement des cours d'eau et de production des milieux aux interfaces du naturel/ culturel et du scientifique/politique.

Pour concevoir cette perspective, nous nous sommes inspirés de l'expression de « connectivité sociale » des cours d'eau, définie par G. Mathias Kondolf et Pedre J. Pinto comme « the communication and movement of people, goods, ideas, and culture along and across rivers, recognizing longitudinal, lateral, and vertical connectivity (la communication et la mobilité des personnes, des marchandises, des idées et de la culture le long des rivières et à travers celles-ci, en distinguant la connectivité longitudinale, latérale et verticale ${ }^{3}$ ) (Kondolf et Pinto, 2017). Cette forme de connectivité comprend ainsi un espace plus large que celui considéré dans la CECE en s'intéressant aux échanges socionaturels et aux usages dans le lit majeur d'un cours d'eau. De manière à outrepasser le seul pourtour d'un cours d'eau et d'intégrer les dimensions bio-géo-physiques, sociopolitiques et sociotechniques, il s'agit de gouverner cette connectivité territoriale qui repose sur un territoire plus labile, qui transgresse ces découpages administratifs.

Appliquer la CECE en matière de connectivité territoriale implique donc l'élargissement de l'espace de discussion jusqu'ici confiné aux seuls propriétaires d'ouvrages et de prendre en considération les interconnexions des échelles et des niveaux de décision. Agir au nom d'une connectivité territoriale identitaire et stratégique permettrait de mieux intégrer le passé (des usages, des aménagements), le présent (état actuel du sociomilieu) et le futur (projet à composer) pour produire un paysage de l'eau comme un «objet vivant construit» (Micoud, 2000). Néanmoins, la formulation de cette proposition n'a pas suffisamment intéressé les gestionnaires locaux qui ont piloté les opérations étudiées. Le contexte difficile d'application de la politique publique à l'échelle nationale et le climat de défiance généralisée n'ont pas permis d'accueillir sereinement cette proposition et de la mettre en pratique pour le moment.

\section{Conclusion}

La politique de la CECE est une politique publique ambitieuse dans ses intentions qui bute contre la faible place accordée aux dimensions territoriales. La naturalisation des continuités des cours d'eau, au détriment de leurs histoires hydrosociales complexes, se caractérise par une approche spatiale qui circonscrit les enjeux aux seuls poissons migrateurs. Conçue essentiellement à partir de savoirs issus de la biologie et de l'hydromorphologie, la CECE a provoqué, sur certains territoires, des conflits, stimulés par l'activité de terrain des propriétaires d'ouvrages hydrauliques et de riverains attachés au patrimoine bâti et/ou à leurs usages du cours d'eau. Ces propriétaires sont décidés à orienter les projets initiaux pour les conformer à l'identité qu'ils se font des eaux de leurs territoires. Dans ce but, ils entendent sortir d'une logique sectorielle (préservation de la biodiversité) pour donner vie à la formulation de projets de territoire. 
Alors même que les outils de cette action publique s'intéressent assez peu aux paysages dans leurs aspects aussi bien esthétiques que fonctionnels, nous avons présenté la multiplicité des discours des acteurs sur les usages et les savoirs. Cette mise en exergue a permis de montrer les différences fondamentales des projets de territoires, en relation avec les eaux, défendus par les acteurs. En explicitant les éléments des savoirs relatifs aux paysages, nous avons identifié une voie pour penser les fins et les moyens des actions relatives à la CECE et limiter les asymétries. L'entrée paysagère des territoires par les savoirs permettrait de ne pas faire du savoir un outil de domination mais un moyen pour discuter de la production d'un empaysagement du territoire.

\section{BIBLIOGRAPHIE}

Alexandre, O. et Arrus, R., « Les « territoires » de l'eau », Cybergeo. Revue européenne de géographie, novembre 2004, URL : http://journals.openedition.org/cybergeo/1249, DOI : 10.4000/cybergeo. 1249.

Babou, I., "Patrimonialisation et politiques de la nature : le parc national de La Réunion », VertigO. La revue électronique en sciences de l'environnement, vol. 15, $\mathrm{n}^{\circ} 1$, mai 2015, URL : URL : http://journals.openedition.org/vertigo/16038; DOI : 10.4000/vertigo.16038.

Barbier, R. et Larrue, C., « Démocratie environnementale et territoires : un bilan d'étape », Participations, $\mathrm{n}^{\circ}$ 1, 2011, p. 67-104.

Barraud, R., « Vers un "tiers-paysage” ? Géographie paysagère des fonds de vallées sudarmoricaines. Héritage, évolution, adaptation », thèse de géographie, université de Nantes, 2007.

Barraud, R., « Rivières du futur, wild rivers? ", VertigO. La revue électronique en sciences de l'environnement, hors-série 10, décembre 2011, URL : http://journals.openedition.org/vertigo/ 11411 ; DOI : $10.4000 /$ vertigo.11411.

Battais, M., Chevallier, K., Elli, A., Faburel, G. (coord.), « Les paysages, les milieux écologiques et l'environnement vécu comme outils dialogiques d'analyse et de prospective territoriale pour l'implantation d'équipements structurants », communication lors du $1^{\text {er }}$ colloque international du CIST « Fonder les sciences du territoire », 2011, p. 29.

Bercovitz, R., « Paysage, médiation paysagère et "bon état écologique" de la haute vallée de la Sèvre niortaise : mener une enquête historique pour fonder un projet partagé (XVIIIe XXI ${ }^{\mathrm{e}}$ siècles) ", thèse de géographie, université Bordeaux III, 2016.

Bertrand, G., « La science du paysage, une science diagonale », Revue géographique des Pyrénées et du Sud-Ouest, T. 43, fascicule II, 1972, p. 127-134.

Blanchon, D., L'Espace hydraulique sud-africain, le partage des eaux, Paris, Karthala, 2009, 294 p.

Bouleau, G. et Pont, D., « Les conditions de référence de la directive-cadre européenne sur l'eau face à la dynamique des hydrosystèmes et des usages », Natures Sciences Sociétés, vol. 22, 2014. 
Coninck, A. de, « Faire de l'action publique une action collective : Expertise et concertation pour la mise en œuvre des continuités écologiques sur les rivières périurbaines », thèse en urbanisme, université Paris-Est, 2015, 750 p.

Davodeau, H. et Romain, F., «L'intégration de la rivière restaurée dans un projet de paysage urbain : une alternative pour penser et agir sur la restauration écologique des cours d'eau? ", dans Germaine, M.-A. et Barraud, R. (dir.), Démanteler les barrages pour restaurer les cours d'eau. Controverses et représentations, Versailles, éditions Quae, 2017.

Desrosières, A., « Le territoire et la localité : deux langages statistiques », Politix, n² 25, 1994.

Dufour, S., Rollet, A. J., Chapuis, M., Provansal, M., Capanni, R., « On the political roles of freshwater science in studying dam and weir removal policies : A critical physical geography approach », Water Alternatives, vol. 10, n 3, 2017, p. 853-869.

Germaine, M.-A., « Apport de l'analyse de discours pour renseigner les représentations paysagères et les demandes d'environnement. Exemple des vallées du nord-ouest de la France ", Annales de géographie, $\mathrm{n}^{\circ}$ 682, 2011, p. 629-650.

Germaine, M.-A., « De la caractérisation à la gestion des paysages ordinaires des vallées du nordouest de la France. Représentations, enjeux d'environnement et politiques publiques en BasseNormandie ", thèse de géographie, université de Caen, 2009, 646 p.

Germaine, M.-A. et Barreaux, R. (coord.), Démanteler les barrages pour restaurer les cours d'eau. Controverses et représentations, Versailles, éditions Quae, 2017, 260 p.

Germaine, M.-A. et Barraud, R., « Les rivières de l'ouest de la France sont-elles seulement des infrastructures naturelles? Les modèles de gestion à l'épreuve de la directive-cadre sur l'eau ", Natures Sciences et société, $\mathrm{n}^{\circ}$ 4, vol. 21, 2013.

Germaine, M.-A. et Lespez, L., « Le démantèlement des barrages de la Sélune (Manche). Des réseaux d'acteurs au projet de territoire ? ", Développement durable et territoires, $\mathrm{n}^{\circ}$ 3, vol. 5, 2014.

Ghiotti, S., « Les Territoires de l'eau et la décentralisation. La gouvernance de bassin-versant ou les limites d'une évidence ", Développement durable et territoires, $\mathrm{n}^{\circ}$ 6, 2006.

Joly, P.-B., «La fabrique de l'expertise scientifique : contribution des STS », Hermès, La Revue, $\mathrm{n}^{\circ} 64,2012$, p. 22-28.

Kondolf, M. G. and Pinto, P. J., « The Social Connectivity of Urban Rivers », Geomorphology, vol. 277, 2017, p. 186-196.

Larrère, C. et Larrère, R. (dir.), La Crise environnementale, Versailles, Inra Éditions, coll. « Les Colloques », 1997, $302 \mathrm{p}$.

Le Calvez, C., « Les usagers confrontés à la restauration de la continuité écologique des cours d'eau : approche en région Bretagne ", thèse de géographie, université de Rennes 2, 2017, 436 p.

Lenclud, G., « La tradition n'est plus ce qu'elle était... Sur la notion de "tradition" et de "société traditionnelle" en ethnologie », Terrain, $n^{\circ}$ 9, 1987, p. 110-123.

Massardier, G., « La gouvernance de l'eau : entre procédure de concertation et régulation adhocratique. Le cas de la gestion de la rivière Verdon en France ", Vertigo. La revue électronique en sciences de l'environnement, hors série 6, novembre 2009, URL : http://journals.openedition.org/ vertigo/8993; DOI : 10.4000/vertigo.8993.

Micoud, A., « Patrimonialiser le vivant », Espaces Temps, n 74-75, 2000, p. 66-77. 
Perrin, J-A., « Gouverner les cours d'eau par un concept : analyse critique de la continuité écologique et de ses traductions, thèse de géographie ", université de Limoges, 2018, 364 p.

Reyt, P., «Formes et paysages de l'eau dans le bassin de la Loire : de l'eau dans le paysage aux paysages de l'eau », thèse de géographie, université de Saint-Étienne, 1997.

Rivière-Honegger, A., « Évolution des usages de l'eau, dynamiques du territoire et mutations paysagères ", Habilitation à diriger des recherches, 2008, 317 p.

\section{NOTES}

1. Extrait d'entretien avec des agents d'une collectivité territoriale qui pilote une opération de la CECE.

2. Les associations de propriétaires de moulins, de micro- et pico-hydroélectricité, des scientifiques académiques ou gestionnaires s'insurgent depuis dix ans, dans la presse ou des instances officielles, contre la manière d'appliquer la CECE.

3. Traduit par l'auteur.

\section{RÉSUMÉS}

Cet article porte son attention sur les effets de l'application d'une politique publique environnementale, appelée continuité écologique des cours d'eau, sur les territoires de l'eau. Mise en œuvre depuis le début des années 2010, cette politique ambitionne de créer des conditions favorables pour la circulation des poissons migrateurs et des sédiments. Conflictuelle lors de certaines opérations, elle fait intervenir de multiples enjeux aux dimensions politiques et scientifiques. Suite à une étude effectuée entre 2014 et 2017 en suivant des opérations de continuité écologique dans les bassins-versants de la Dordogne et de la Têt, nous analysons les conséquences de ce type d'opérations sur les paysages et usages de l'eau avec des modifications parfois importantes dans le cas de la destruction d'ouvrages hydrauliques. Compte tenu de la manière dont sont appréhendés les enjeux paysagers dans la gestion de cette politique, son actuel cadrage ne permet pas de sortir d'une logique de gestion sectorielle centrée sur la biodiversité. Dans ce but, à partir des observations de terrain, nous formulons une proposition axée sur les savoirs liés aux paysages pour parvenir à appliquer cette politique publique en tant que gouvernement d'un territoire de l'eau.

This article focuses on the effects on waterscapes of the application of a public environmental policy referred to as the ecological continuity of waterscapes. Implemented since the beginning of 2010 , this policy is intended to create favourable conditions for the circulation of migratory fish and sediments. Generating conflicts in the case of certain operations, it involves multiple issues encompassing political and scientific dimensions. Following a study carried out between 2014 and 2017 monitoring operations of ecological continuity in the Dordogne and Têt catchment areas, we analysed the consequences of this type of operation on landscapes and on water uses, which sometimes involve significant modifications in the case of the destruction of hydraulic works. Given how landscape issues are addressed in the implementation of this policy, its current framework does not make it possible to depart from a sector-based management approach 
focused on biodiversity. To this end, using field observations, we put forward a proposal focused on landscape-related knowledge as a method for implementing this public policy governing waterscapes.

INDEX

Keywords : conflict, ecological continuity, waterscape, public policy, use

Mots-clés : conflit, continuité écologique, paysage de l'eau, politique publique, usage

\section{AUTEUR}

\section{JACQUES-ARISTIDE PERRIN}

Postdoctorant en géographie, GEOLAB, université de Limoges, Jacques-Aristide Perrin s'intéresse aux rôles des savoirs dans le gouvernement des cours d'eau.

jacques-aristide.perrin[at]etu.unilim[dot]fr 